\title{
Atividade física para crianças e jovens: Guia de Atividade Física para a População Brasileira
}

\section{Physical activity for children and youth: Physical Activity Guidelines for the Brazilian Population}

\section{AUTORES \\ Samuel Carvalho Dumith ${ }^{1}$ (D) \\ Alcides Prazeres Filho' ${ }^{2}$ D \\ Felipe Vogt Cureau ${ }^{3}$ (iD \\ José Cazuza de Farias Júnior ${ }^{2,4}$ (D) \\ Júlio Brugnara Mello ${ }^{5}$ (D) \\ Michael Pereira da Silva ${ }^{6}$ (iD \\ Thiago Sousa Matias ${ }^{7}$ (D) \\ Wendell Arthur Lopes ${ }^{8}$ (iD \\ Lorena Lima Magalhães ${ }^{9}$ (D) \\ Pedro Curi Hallal ${ }^{10}$ (D) \\ 1 Universidade Federal do Rio Grande, Programa de Pós-graduacão em Ciências da Saúde, Rio Grande, Rio Grande do Sul, Brasil. \\ 2 Universidade Federal da Paraíba, Departamento de Educação Física, João Pessoa, Paraíba, Brasil. \\ 3 Universidade Federal do Rio Grande do Sul, Programa de Pós-Graduação em Ciências da Saúde: Cardiologia e Ciências Cardiovasculares, Porto Alegre, Rio Grande do Sul, Brasil. \\ 4 Programa Associado de Pós-Graduação em Educação Física - Universidade de Pernambuco/ Universidade Federal da Paraíba, João Pessoa, Paraíba, Brasil. \\ 5 Faculdade SOGIPA, Departamento de Educação Física, Porto Alegre, Rio Grande do Sul, Brasil \\ 6 Universidade Federal do Rio Grande, Faculdade de Medicina, Rio Grande, Rio Grande do Sul, Brasil \\ 7 Universidade Federal de Santa Catarina, \\ Departamento de Educação Física, Florianópolis, \\ Santa Catarina, Brasil \\ 8 Universidade Estadual de Maringá, Departamento de Educação Física, Maringá - Paraná - Brasil. \\ 9 Ministério da Saúde. Brasília, DF, Brasil. \\ 10 Universidade Federal de Pelotas, Departamento de Ginástica e Saúde, Pelotas, Rio Grande do Sul, Brasil.}

\section{CONTATO}

Samuel Carvalho Dumith

scdumith@yahoo.com.br

Rua Visconde de Paranaguá, 102, Centro, Rio Grande, Rio Grande do Sul, Brasil.

CEP: 96200-190.

$\mathrm{DOI}$

$10.12820 /$ rbafs.26e0214

\begin{abstract}
RESUMO
A prática regular de atividade física promove benefícios fisiológicos, psicológicos e sociais na vida de crianças e jovens. Apesar da alta prevalência de jovens que não praticam atividade física em nosso país e de termos uma boa colocação no ranking mundial de produção acadêmica sobre pesquisa na área de atividade física e saúde, ainda não havia sido publicadas as recomendações brasileiras de atividade física. O objetivo deste artigo consistiu em descrever como foi desenvolvido o capítulo para crianças e jovens de 6 a 17 anos do Guia de Atividade Física para População Brasileira e apresentar as principais recomendações para esta faixa etária. A construção do capítulo para crianças e jovens envolveu as seguintes etapas: a) revisão de literatura; b) redação da versão preliminar do capítulo; c) processo de escuta com o público alvo referente ao capítulo e especialistas da área de promoção da atividade física; d) realização de consulta pública e; e) redação da versão final do capítulo. Os tópicos apresentados abordam exemplos de atividades físicas praticadas em diferentes domínios; recomendações para a prática (tipos, intensidade, frequência, duração e as formas que a mesma pode ser estruturada). São apresentadas orientações para jovens, pais/responsáveis e professores sobre como adotar e manter um estilo de vida mais ativo fisicamente, assim como sugestões para reduzir o tempo em comportamento sedentário. A elaboração de um guia nacional de atividade física para a população brasileira foi algo inédito, e resultou de um esforço conjunto entre diversos setores da sociedade.
\end{abstract}

Palavras-chave: Atividade física; Promoção da saúde; Políticas públicas; Criança; Adolescente.

\section{ABSTRACT}

Regular physical activity promotes physiological, psychological and social benefits in the lives of children and adolescents. Despite the high prevalence of young people who do not physically activity in our country, and that we have a good ranking in world academic production on research in the physical activity and health area, Brazilian recommendations for physical activity had not been published until then. The aim of this article was to describe how the chapter for children and young people aged 6-17 years of Physical Activity Guidelines for the Brazilian Population was developed and to present the main recommendations for this age group. The construction of the chapter for children and young people involved the following steps: a) literature review; b) writing the preliminary version of the chapter; c) listening process of the target audience for the chapter and specialists in the physical activity promoting area; d) public consultation; and e) writing the final version of the chapter. The topics presented cover examples of physical activities practised in different domains; recommendations for practice (types, intensity, frequency, duration and the ways in which it can be structured). Guidance is provided for young people, parents/guardians and teachers about how to adopt and maintain a more physically active lifestyle, as well as suggestions for reducing time in sedentary behaviour. The construction of a national guidelines about physical activity for Brazilian population is something unprecedented, and resulted from a joint effort of several departments of society.

Keywords: Physical activity; Health promotion; Public health; Child; Adolescent.

\section{(c) BY}




\section{Introdução}

A atividade física é um comportamento que promove uma série de benefícios fisiológicos, psicológicos, sociais e apropriações culturais na vida da criança e do jovem ${ }^{1}$. A realização apropriada de atividade física pode favorecer aspectos de saúde como a melhora dos parâmetros da aptidão física e desenvolvimento motor, manutenção do peso corporal, prevenção de doenças crônicas não transmissíveis (DCNT), manutenção e promoção da saúde mental, além de favorecer a interação social desse público ${ }^{2,3}$. A manutenção deste comportamento nesta fase da vida favorece a adoção de outros comportamentos positivos de saúde como diminuição do tempo em comportamentos sedentários, alimentação mais equilibrada e saudáve $1^{4}$ e diminuição na exposição aos comportamentos de riscos como tabagismo, alcoolismo ou uso de drogas ilícitas 5 .

Apesar do conhecimento sobre os benefícios supracitados, em torno de $80 \%$ dos adolescentes globalmente não atingem um mínimo de 60 minutos por dia de prática de atividade física ${ }^{6}$. Dados mais recentes reforçam a alta prevalência de inatividade física entre adolescentes e demonstram que este cenário permanece inalterado desde $2001^{7}$. No Brasil, dados da última Pesquisa Nacional de Saúde do Escolar (PeNSE 2015), apontaram que $71,7 \%$ dos meninos e $86,5 \%$ das meninas não particam pelo menos 300 minutos de atividade física por semana ${ }^{8}$.

$\mathrm{Na}$ perspectiva de melhorar o monitoramento e subsidiar políticas para a promoção da atividade física, recomendações de atividade física têm sido construídas em nível global pela Organização Mundial da Saúde ${ }^{1}$ e em nível nacional por diferentes países ${ }^{9-13}$. De forma geral, estas recomendações indicam que crianças e adolescentes (6 a 17 anos) devem acumular, em média, 60 minutos diários em atividades físicas com intensidade moderada a vigorosa ${ }^{1}$. Algumas recomendações indicam, ainda, que atividades físicas de intensidade leve também devem ser realizadas sempre que possível ao longo do dia ${ }^{9,11-13}$. É indicado que as atividades físicas aeróbias devem ocupar a maior parte do tempo, e atividades visando o fortalecimento muscular e ósseo devem ser realizadas, pelo menos, três vezes por semana ${ }^{9-13}$.

Embora o Brasil desempenhe papel de liderança no cenário global de pesquisa em atividade física, ocupando a quarta posição do ranking mundial de produção acadêmica na área, de acordo com o Global Observatory for Physical Activity ${ }^{14}$, até o momento, não produziu suas próprias recomendações de atividade física. Neste sentido, foi desenvolvido o projeto "Guia de Atividade Física para a População Brasileira" (referido daqui para frente como Guia) com intuito de desenvolver as primeiras recomendações do país para a prática de atividade física. O Guia foi elaborado considerando os ciclos da vida (crianças de 0-5 anos; crianças e jovens de 6-17 anos; adultos de 18-59 anos e idosos de 60 anos ou mais), populações específicas (gestantes e pessoas com deficiência), além dos domínios e contextos nos quais se dá a prática de atividade física no Brasil. O presente artigo teve como propósito descrever como foi desenvolvido o capítulo do Guia para crianças e jovens de 6 a 17 anos e apresentar as principais recomendações e mensagens para promoção da atividade física entre os indivíduos desta faixa etária.

\section{Métodos}

Este manuscrito aborda os procedimentos adotados pelo Grupo de Trabalho responsável pela construção do capítulo destinado a crianças e jovens de 6 a 17 anos de idade, do Guia. O trabalho foi estruturado de acordo com os seguintes passos: 1) revisão da literatura e organização da base teórica acerca das principais recomendações de atividade física para crianças e adolescentes no mundo; 2) processo de escuta com o público-alvo (adolescentes, pais/responsáveis e professores); 3) processo de escuta com especialistas da área de promoção da atividade física; 4) redação da versão preliminar do texto que comporia o Guia; 5) disponibilização para a sociedade da primeira versão do texto do capítulo por meio de consulta pública; 6) redação da versão final do capítulo do Guia.

Visando construir uma base de referências bibliográficas para redação do capítulo destinado a crianças e jovens do Guia foi realizada, primeiramente, uma busca por outras recomendações e guias de atividade física que apresentassem alguma seção destinada à população jovem. Sites de diferentes instituições e bases eletrônicas de dados foram consultados. Ao todo, foram selecionados 18 guias/recomendações para prática de atividade física, oriundos de diferentes países e publicados entre janeiro de 2010 e junho de 2020. Os documentos encontrados foram utilizados para subsidiar as recomendações de tempo, intensidades e frequências mínimas para obtenção de benefícios para saúde geral dos jovens.

As bases de dados eletrônicas PubMed, Cocbrane Library e LILACS foram consultadas com intuito de realizar uma investigação sistemática de estudos de revisão sistemática e metanálises sobre intervenção em 
atividade física e comportamento sedentário envolvendo crianças e jovens. Uma outra busca nas mesmas bases de dados foi realizada para seleção de revisões sistemáticas sobre motivações, facilitadores ou barreiras para a prática de atividade física entre crianças e jovens. Depois de finalizado o processo de seleção, somaram-se aos resultados dessas buscas os estudos selecionados a partir de outra estratégia de pesquisa realizada de forma independente pelo Grupo de Trabalho de Domínios da Atividade Física, a qual incluiu 64 estudos.

Os estudos elegíveis para compor o referencial teórico deveriam apresentar os seguintes aspectos: 1 ) ser artigos de revisão sistemática; 2) incluir estudos com jovens entre 6 e 17 anos de idade; 3) abordar questões de interesse deste Grupo de Trabalho, entre elas, intervenções visando aumentar a prática de atividade física e/ou reduzir o tempo em comportamento sedentário, benefícios da prática de atividade física, motivações, facilitadores ou barreiras para prática de atividade física em diferentes contextos; 4) ter sido publicado nos últimos dez anos; 5) ser publicado em português, espanhol ou inglês.

O fluxograma completo do processo de seleção de base de referências bibliográficas está apresentado na Figura 1. Cabe ressaltar que a intenção desta busca não foi realizar uma revisão sistemática de revisões sistemáticas (overview), mas identificar as evidências recentes e relevantes que serviriam de fonte primária para a construção do capítulo do Guia. O instrumento norteador da estrutura de conteúdos incluídos neste capítulo foi um template (com orientações acerca do formato e tópicos que cada capítulo deveria abranger) elaborado pelo Comitê Científico do Guia em conjunto com a equipe do Ministério da Saúde.

Para a construção do texto específico para crianças e jovens foram realizadas escutas com pais, responsáveis, adolescentes (12 a 17 anos) e professores/profissionais de Educação Física atuantes nos sistemas educacionais público e privado. Essas escutas ocorreram no mês de julho de 2020 e tiveram como objetivo coletar informações referentes à compreensão, formato de apresentação e conteúdos, bem como, sugestões a serem aplicadas ao texto preliminar produzido pelo Grupo de Trabalho responsável pela construção deste capítulo do Guia. As escutas foram realizadas por meio de formulários eletrônicos da ferramenta Google Forms.

Com intuito de atingir respondentes de todas as regiões do país, os links dos formulários foram compartilhados com membros do projeto "Recomendações brasileiras de atividade física" e, também, entre contatos dos membros deste Grupo de Trabalho. Essas pessoas atuaram como disseminadores desses links, favorecendo a participação de pessoas de todas as regiões do país nesta etapa da construção do Guia.

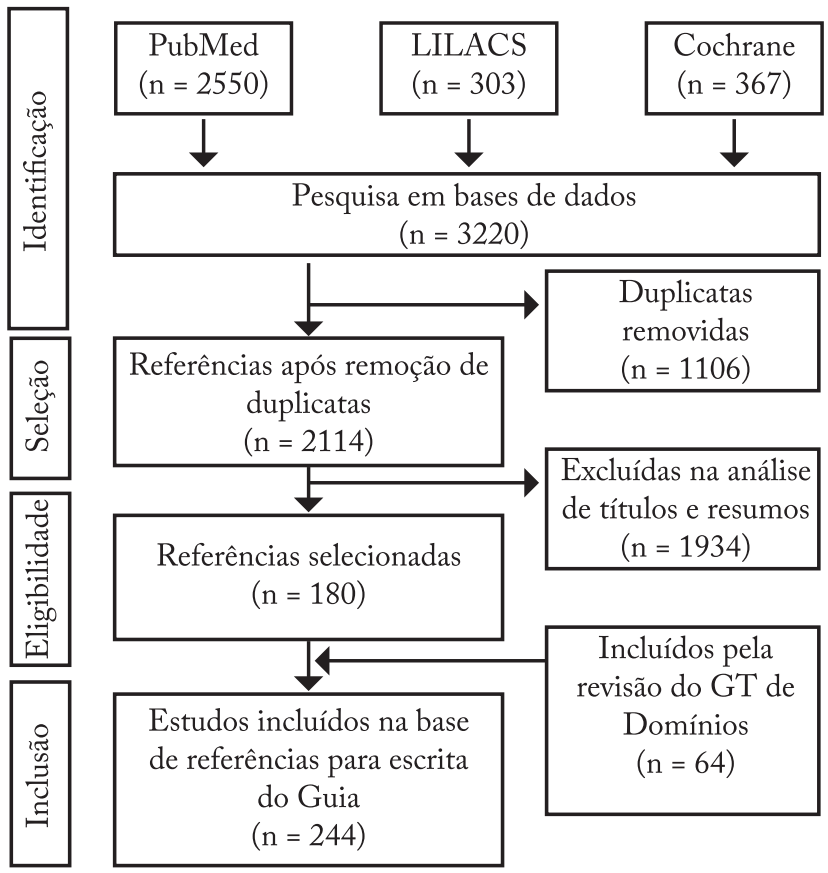

Figura 1 - Fluxograma do processo de seleção da base de referências utilizadas

Como parte adicional a essa escuta, dois especialistas da área de promoção da atividade física, com titulação de doutor, foram entrevistados. Os especialistas possuíam vasta experiência em pesquisas relacionadas às áreas de políticas públicas de esporte e lazer, além de possuirem experiências com a gestão de programas destinados à promoção da prática de atividade física em crianças e adolescentes como, por exemplo o projeto "Segundo Tempo". Também disso, foram consideradas sugestões de outros especialistas da área ouvidos em seminários conduzidos por outros grupos de trabalho, fazendo-se a devida adequação à população jovem.

As entrevistas ocorreram por videoconferências, em julho de 2020, e foram conduzidas por um dos membros do Grupo de Trabalho. As entrevistas foram gravadas e posteriormente utilizadas com intuito de obter informações sobre os sistemas públicos de apoio à promoção de atividade física para crianças e adolescentes no Brasil, bem como a opinião dos especialistas sobre informações que deveriam ser incluídas no Guia. Adicionalmente, com o objetivo de mapear programas e estratégias que promovessem a prática de atividade física para crianças e jovens, foram realizadas buscas em 
sites de Ministérios e Secretarias do Governo Federal.

O Grupo de Trabalho responsável pelas recomendações de atividades físicas para crianças e jovens organizou-se a partir de reuniões semanais com pautas e tarefas previamente definidas por e-mail. Dessa forma, foram organizados dois subgrupos, com três e quatro integrantes, para realizar o levantamento de informações e escrita de um texto sobre: 1) recomendações de atividade física para crianças e adolescentes e prevalência de (in)atividade física a nível nacional e global e; 2) efeitos da atividade física regular, do baixo nível de atividade física e do elevado tempo em comportamento sedentário para saúde de crianças e adolescentes.

Após a criação destes dois primeiros textos, o Grupo de Trabalho destinou seus esforços para a construção da versão preliminar do capítulo. Posteriormente, foram incorporados aspectos relevantes levantados nas escutas públicas e com especialistas à versão preliminar do capítulo. Como parte da proposta de criação do Guia, o Ministério da Saúde, por meio da Secretaria de Atenção Primária à Saúde realizou, no mês de agosto de 2020, a Consulta Pública ao documento completo construído pelos documentos dos oito Grupos de Trabalho (vide notícia em: https://aps.saude.gov.br/noticia/9516). Após o término dessa consulta, as sugestões e observações obtidas foram analisadas pelos membros do Grupo de Trabalho e incorporadas ao documento de forma a integrar a participação da sociedade brasileira na construção do Guia.

O Guia, em sua sessão dedicada a crianças e jovens (6 a 17 anos), traz orientações simples e em linguagem acessível, fisicamente a fim de auxiliar o público-alvo na adoção de um estilo de vida mais ativo. São apresentadas recomendações quanto ao volume e intensidade de atividade física necessários para obtenção de benefícios para saúde, de que forma o volume recomendado pode ser acumulado ao longo do dia, exemplos de atividades físicas que podem ser praticadas nos diferentes domínios e orientações sobre a prática direcionadas aos jovens. Pais, responsáveis e professores de Educação Física também receberam orientações a fim de que possam influenciar positivamente e dar suporte aos jovens na realização da prática de atividade física.

\section{Resultados}

Os resultados aqui delimitados são uma síntese dos elementos centrais que sustentam cientificamente as orientações sobre atividades físicas para crianças e jovens. O conteúdo completo do Guia de Atividade
Física para a População Brasileira pode ser acessado no site do Ministério da Saúde (www.saude.gov.br). Alguns exemplos de atividades físicas que podem ser praticadas, nos diferentes domínios, estão descritas no Quadro 1. Estes exemplos tem por objetivo reforçar que as crianças e jovens podem ser fisicamente ativos em diferentes domínios e não apenas durante o tempo livre (lazer).

Quadro 1 - Domínios e respectivos exemplos de atividades físicas para jovens (6-17 anos).

\begin{tabular}{|c|c|c|}
\hline \multirow{4}{*}{$\begin{array}{l}\text { Domínios } \\
\text { da atividade } \\
\text { física }\end{array}$} & $\begin{array}{l}\text { No tempo } \\
\text { livre (lazer) }\end{array}$ & $\begin{array}{l}\text { Esportes individuais e coletivos: futebol, } \\
\text { futsal, handebol, vôlei, basquete, rúgbi, } \\
\text { esportes com raquete, ginástica rítmica ou } \\
\text { artística, atletismo, artes marciais. } \\
\text { Atividades individuais e em grupos: } \\
\text { caminhar, correr, empinar pipa, dançar, } \\
\text { nadar, pedalar, andar de skate, brincadeiras } \\
\text { e jogos (esconde-esconde, pega-pega, pular } \\
\text { corda, saltar elástico queimada/baleado/ } \\
\text { carimba/caçador). }\end{array}$ \\
\hline & $\begin{array}{l}\text { No } \\
\text { deslocamento }\end{array}$ & $\begin{array}{l}\text { Fazer deslocamentos a pé ou de bicicleta, } \\
\text { de skate, de patins ou de patinete (sem } \\
\text { motor). }\end{array}$ \\
\hline & No estudo & $\begin{array}{l}\text { Participar das aulas de Educação Física, } \\
\text { de brincadeiras e jogos, das equipes } \\
\text { esportivas da escola, das atividades físicas } \\
\text { extracurriculares e daquelas que ocorrem } \\
\text { nos espaços livres da escola durante o } \\
\text { recreio/intervalo. } \\
\end{array}$ \\
\hline & $\begin{array}{l}\text { Nas tarefas } \\
\text { domésticas }\end{array}$ & $\begin{array}{l}\text { Ajudar seus familiares em algumas tarefas } \\
\text { da casa como conservação do seu quintal, } \\
\text { fazer jardinagem, lavar a bicicleta ou dar } \\
\text { banho no animal de estimação ou recolher } \\
\text { o lixo. }\end{array}$ \\
\hline
\end{tabular}

Para a faixa etária de 6 a 17 anos, as recomendações preconizam que se deve acumular pelo menos 60 minutos por dia em atividade física de intensidade moderada a vigorosa. Essa recomendação pode ser alcançada de forma contínua, em apenas uma sessão, ou acumulada em períodos menores ao longo do dia. Além disso, recomenda-se que os jovens pratiquem atividades físicas de fortalecimento muscular e ósseo em pelo menos três dias ao longo da semana, como parte dos 60 minutos ou mais por dia recomendados (Quadro 2). Para aqueles jovens que, inicialmente, não conseguem atingir os $60 \mathrm{minu}-$ tos diários de atividade física, ressalta-se a importância de acumular a maior quantidade de minutos de prática possível e, com um aumento de volume progressivo ao longo do tempo, buscando atingir as recomendações.

O Guia de Atividade Física para a População Brasileira também apresenta orientações aos jovens, pais/ responsáveis e professores de Educação Física sobre como os jovens podem adotar um estilo de vida mais 
Quadro 2-Recomendações de prática de atividade física para crianças e jovens de 6 a 17 anos

\begin{tabular}{|l|l|l|}
\hline \multirow{4}{*}{ Recomendações } & Tipo & $\begin{array}{l}\text { Atividades físicas em geral, incluindo } \\
\text { atividades aeróbicas, de fortalecimento } \\
\text { muscular e ósseo* }\end{array}$ \\
\cline { 2 - 3 } & Intensidade & Moderada a vigorosa** \\
\cline { 2 - 3 } & Frequência & Todos os dias da semana \\
\cline { 2 - 3 } & Duração & 60 minutos por dia \\
\cline { 2 - 3 } & $\begin{array}{l}\text { Estruturação } \\
\text { da prática }\end{array}$ & Contínua e/ou acumulada*** \\
\hline
\end{tabular}

*Como parte dos 60 minutos de prática; ${ }^{* *}$ Atividades com intensidade absoluta superior a 3 METs, ou seja, aquelas que levam a um aumento moderado da frequência cardíaca e respiratória; ***Contínua: 60 minutos por dia em uma única sessão; acumulada: 60 minutos por dia fracionado em blocos de tempo ao longo do dia.

ativo fisicamente, superar eventuais barreiras para prática de atividade física e reduzir o tempo em comportamento sedentário (Quadro 3). Para as crianças e jovens, as mensagens objetivam estimular a prática de atividade física nos diferentes domínios, e incentivar a experimentação de diferentes tipos de atividade física, estimulando que façam algo que gostem. Foi reforçado que $\mathrm{a}$ iniciação às atividades físicas poderia acontecer a qualquer momento e sugerido incluir a atividade física como parte da rotina diária das criaças e jovens. Uma das mensagens do Guia destaca a importância de envolver amigos e familiares (prática conjunta) na condução das atividades físicas.

Para os pais ou responsáveis, as principais mensagens foram direcionadas ao reconhecimento da importância do apoio deles para a prática de atividade física dos filhos, sobretudo das crianças, que têm maior dependência da companhia, permissão e/ou acompanhamento no deslocamento até os locais de prática. As mensagens para os professores de Educação Física destacaram o importante papel deles como modelo de comportamento para seus alunos. Foi destacado o papel das aulas de Educação Física para o incentivo e cumprimento das recomendações de atividade física, e sugerido que os professores abordem os conteúdos do Guia em consonância com as competências e habilidades destacadas na Base Nacional Comum Curricular (BNCC) - Quadro 3.

Orientações para reduzir o tempo em comportamento sedentário também foram contempladas. Reforçou-se a importância de limitar o tempo de tela diário no tempo livre em no máximo duas horas e substituir o tempo em comportamento sedentário por alguma atividade física. Realizar pausas durante períodos prolongados em comportamento sedentário é algo que deve ser
Quadro 3-Síntese das principais mensagens e orientações sobre como adotar um estilo de vida mais ativo fisicamente

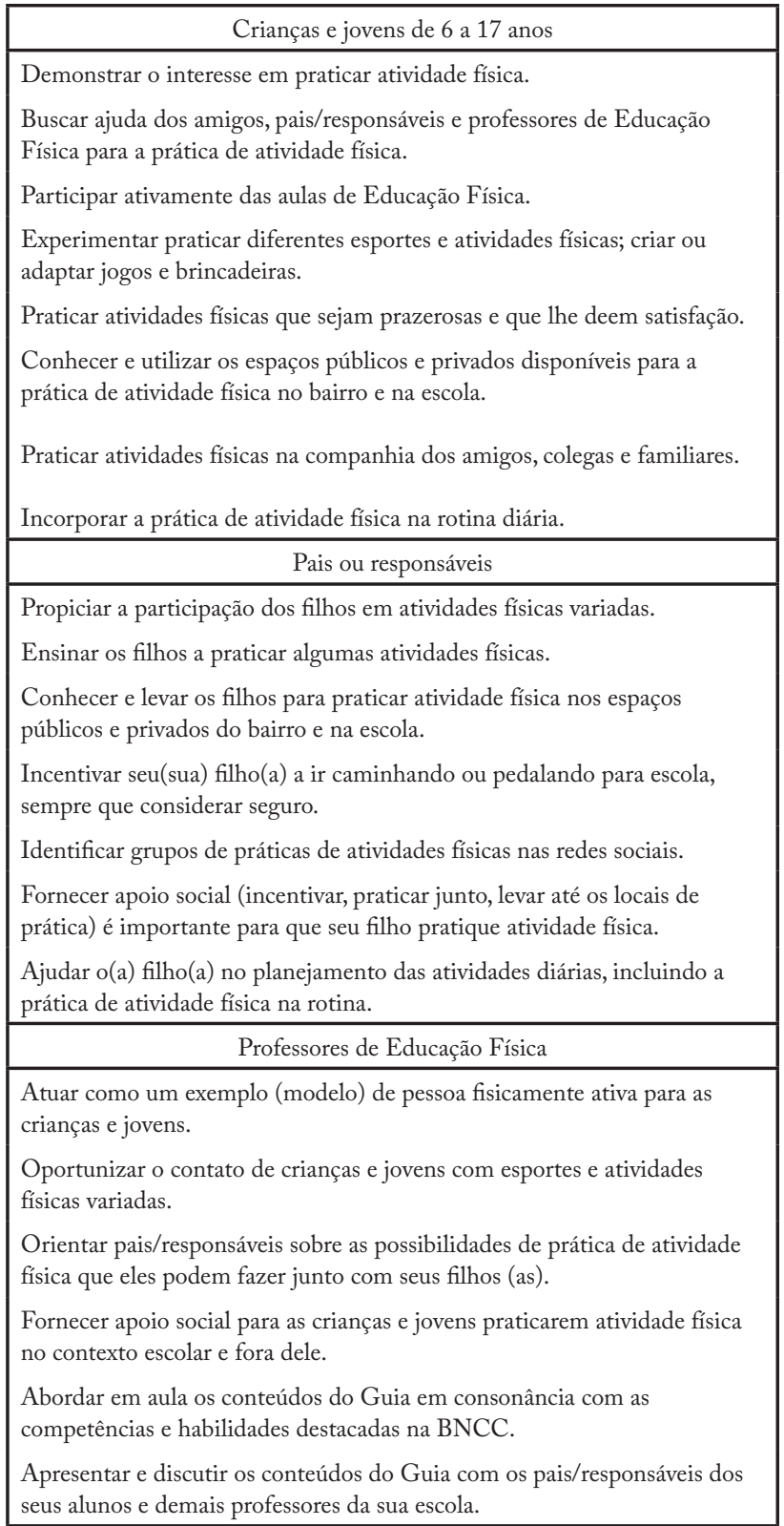

incentivado. $\mathrm{Na}$ escola, os professores de Educação Física devem propor momentos ativos durante o período escolar (recreio/intervalo, atividades extra-classe), além de buscar a inclusão transversal dessa temática no projeto político pedagógico das escolas (Quadro 4). Uma das recomendações do Grupo de Trabalho para diminuir o comportamento sedentário é que as crianças e jovens assistam parte das aulas em pé na escola, com o auxílio de mesas ajustáveis, tal como já é feito em outros países.

\section{Discussão}

O presente artigo apresentou as estratégias e métodos 
Quadro 4-Síntese das principais mensagens e orientações sobre como reduzir o tempo em comportamento sedentário.

\begin{tabular}{|c|}
\hline Crianças e jovens (6 a 17 anos) \\
\hline $\begin{array}{l}\text { Limitar em, no máximo, duas horas por dia, o tempo em frente ao } \\
\text { computador, celular, tablet, videogame e televisão. }\end{array}$ \\
\hline $\begin{array}{l}\text { Substituir parte do tempo em comportamentos sedentários pela prática de } \\
\text { alguma atividade física. }\end{array}$ \\
\hline $\begin{array}{l}\text { Incluir pausas durante a realização das tarefas da escola: levantar, se } \\
\text { alongar, mexer o seu corpo ou caminhar para tomar um copo de água, por } \\
\text { exemplo. }\end{array}$ \\
\hline Pais ou responsáveis \\
\hline $\begin{array}{l}\text { Limitar em, no máximo, duas horas por dia o tempo em que seu(sua) } \\
\text { filho(a) passa em frente ao computador, celular, tablet, videogame e televisão. }\end{array}$ \\
\hline $\begin{array}{l}\text { Estimular seu(sua) filho(a) a substituir uma parte do tempo livre em } \\
\text { comportamento sedentário por jogos ou brincadeiras ativas. }\end{array}$ \\
\hline $\begin{array}{l}\text { Orientar o(a) filho(a) para fazer uma pausa, a cada uma hora sentado, para } \\
\text { se levantar, se alongar, mexer o corpo ou caminhar para tomar um copo } \\
\text { de água. }\end{array}$ \\
\hline Professores de Educação Física \\
\hline $\begin{array}{l}\text { Incluir na rotina escolar jogos e atividades que estimulem os alunos a } \\
\text { diminuírem o tempo em comportamento sedentário. }\end{array}$ \\
\hline $\begin{array}{l}\text { Discutir com os demais professores da escola sobre "comportamentos } \\
\text { sedentários" e como limitar o tempo em que os jovens passam nesses } \\
\text { comportamentos. }\end{array}$ \\
\hline $\begin{array}{l}\text { Sugerir que o tópico “comportamentos sedentários” seja incluído como } \\
\text { tema transversal no projeto político-pedagógico da sua escola. }\end{array}$ \\
\hline
\end{tabular}

adotados para construção do Guia de Atividade Física para População Brasileira, bem como as principais informações presentes na seção relacionada às crianças e jovens de 6 a 17 anos de idade. O Guia representa um esforço conjunto entre o Estado, a comunidade científica e a sociedade em geral na construção de um documento que será utilizado como importante fonte de informações para a população geral, para profissionais de saúde e para responsáveis pela criação e implementação de políticas públicas de promoção da atividade física em diferentes ciclos de vida, contextos e condições específicas. Para as crianças e jovens brasileiros de 6 a 17 anos, o Guia recomenda a prática de, em média, 60 minutos diários de atividades físicas de moderada à vigorosa intensidade, incluindo atividades aeróbias e de fortalecimento muscular e ósseo.

Apesar da existência de recomendações globais de atividade física e comportamento sedentário ${ }^{15}$, diversos países propuseram seus próprios guias nacionais com o intuito de atender características específicas de sua população e fornecer subsídios específicos para a promoção de atividade física, considerando as suas particularidades. Dentre esses países destacam-se os Estados Unidos da América $^{3}$, o Canadá ${ }^{16}$, a Austrália ${ }^{9}$, o Reino Unido ${ }^{17}$, a China $^{10}$, o Uruguai ${ }^{18}$, a Argentina ${ }^{19}$, entre outros ${ }^{20-23}$.

Em termos gerais, o Guia acompanhou as recomendações para a prática de atividade física (por exemplo: tipos, volume e intensidade de prática de atividade física) presentes nos guias de outros países. Porém, cabe destacar o avanço ao incluir mensagens voltadas para pais/responsáveis e professores de Educação Física, apresentando exemplos de atividades físicas que as crianças e os jovens podem praticar em diferentes domínios e levando em consideração as diferentes realidades e particularidades regionais e culturais. Também foram trazidas mensagens de como superar barreiras para a prática e formas de como reduzir o tempo em comportamento sedentário, destinadas as crianças e jovens, seus pais/responsáveis e professores.

Uma recente revisão ${ }^{24}$ sobre as diferentes recomendações de atividade física para crianças e adolescentes mostrou que, assim como o Guia, o volume de 60 minutos diários é um consenso, e corrobora com a recente recomendação global de atividade física proposta pela $\mathrm{OMS}^{15}$. O volume "ótimo" de atividade física é uma delimitação complexa, entretanto, há evidencias de que muitos dos benefícios decorrentes da prática de atividade física ocorrem com volumes em torno de $60 \mathrm{mi}^{-}$ nutos diários ${ }^{15}$.

O Guia, todavia, aborda de forma enfática que o tempo de atividade física pode ser cumulativo ao longo do dia e mesmo que a criança ou adolescente não atinja diariamente essa meta, que ele(a) busque sempre o engajamento em alguma atividade física todos os dias da semana. Tal estratégia foi adotada na tentativa de não desestimular jovens que em alguns dias da semana não cumpram os 60 minutos diários recomendados.

Com relação à intensidade das atividades físicas, os guias internacionais ${ }^{24}$, prioritariamente, recomendam atividades físicas de intensidade moderada a vigorosa. A recomendação de realização de atividades físicas que atinjam intensidades moderada e vigorosa dá-se a partir da associação entre essa faixa de intensidade e os múltiplos benefícios à saúde de crianças e adolescentes tais como, melhora na aptidão cardiorrespiratória, neuromuscular, óssea, de indicadores cardiometabólicos, cognitivos, controle de peso corporal/adiposidade e redução de sintomas depressivos ${ }^{2,15}$. O Guia, além de seguir esta recomendação geral, traz um ponto convergente com a maioria dos guias internacionais: trata da recomendação de prática de atividades físicas de intensidade leve. O Guia sugere que, sempre que possível, seja realizada alguma atividade física, mesmo que de 
intensidade leve, ao longo do dia, como uma forma de reduzir ao máximo o tempo em comportamento sedentário. Essa recomendação baseia-se em evidências que indicam que o tempo em atividade física leve pode aumentar os benefícios gerais de ser fisicamente ativo ${ }^{25}$.

Quanto ao tipo de atividade física recomendada, as atividades físicas aeróbias são a prioridade na maioria das recomendações internacionais para crianças e adolescentes ${ }^{24}$. Tanto a recomendação global proposta pela OMS como os principais guias internacionais recomendam as atividades físicas que envolvam fortalecimento muscular e ósseo por, pelo menos, três vezes por semana. O Guia segue estas recomendações. No entanto, ao descrever os benefícios das atividades físicas recomendadas, além dos benefícios tradicionalmente descritos, considera também que as atividades praticadas tenham impacto positivo no desenvolvimento das habilidades motoras e nas capacidades físicas (por exemplo: flexibilidade, velocidade $)^{26}$.

Com relação ao comportamento sedentário, embora existam evidências sugerindo que o tempo despendido em atividades sedentárias está relacionado a desfechos negativos na saúde dos jovens, como baixa aptidão física, maior adiposidade e piora no perfil cardiometabólico, a quantidade mínima em comportamento sedentário recomendada e o impacto da interrupção do comportamento sedentário ainda não estão bem evidenciados ${ }^{27}$. Dessa forma, o presente Guia recomendou que as crianças e os jovens reduzam e/ou substituam o tempo em comportamento sedentário, sempre que possível, por atividades físicas, mesmo que de baixa intensidade.

Essas recomendações do Guia são direcionadas ao público alvo (crianças e jovens de 6 a 17 anos), mas também às pessoas ligadas a esta faixa etária. Dessa forma, pais ou responsáveis, professores em geral, especialmente os de Educação Física, podem acessar as informações do Guia para atuar como agentes promotores de atividade física para este público. Essa proposta baseia-se no entendimento ecológico da atividade física ${ }^{28} \mathrm{em}$ que não se percebe a criança e o jovem como os únicos responsáveis pela prática de atividade física ou pelo tempo em comportamento sedentário no seu cotidiano.

Nessa perspectiva, o Guia traz informações sobre o apoio social dos pais e responsáveis ${ }^{29}$ e das atividades que podem ser propostas e estimuladas pelos professores, corroborando o que é recomendado especificamente a esta faixa etária ${ }^{26}$. Os agentes promotores da atividade física podem ter acesso às informações rela- cionadas ao uso de espaços públicos e ao engajamento em esportes e atividades no tempo livre ${ }^{30}$ que estão sumarizadas no Guia.

É importante destacar que, apesar de o Guia ser um importante documento para disseminação de informações e recomendações sobre adoção de um estilo de vida fisicamente ativo, este, por si só, não é capaz de promover mudanças em termos de aumento dos níveis de atividade física de crianças e jovens. Para tanto, é necessário que a disseminação das recomendações do Guia esteja articulada às ações de diferentes setores da sociedade (Saúde, Educação, entre outros) e aos programas e políticas de promoção da atividade física nas diferentes esferas (pública e privada) e nos diferentes níveis (municipal, estadual e federal).

A publicação do Guia contribuirá para a promoção da prática de atividade física no Brasil, com implicações positivas na saúde pública da população. Ele estará disponível em linguagem acessível, visando facilitar a compreensão e a disseminação de informações, recomendações e mensagens sobre a adoção de um estilo de vida fisicamente ativo e menos sedentário. As suas mensagens foram direcionadas as crianças e aos jovens, pais/responsáveis e aos professores de Educação Física, pois há o entendimento de que a adoção de um estilo de vida ativo não é uma escolha meramente individual, sendo fundamental uma rede de apoio que crie condições favoráveis para prática e, aqueles mais próximos dos jovens, representam os elos iniciais dessa rede.

Concluindo, o planejamento e a elaboração de um guia de atividade física para a população brasileira foi algo inédito e demandou um esforço conjunto entre diversos setores da sociedade. Isso permitiu a elaboração de um documento coerente com a produção científica atual neste campo do conhecimento e com as necessidades da população brasileira. Assim como outras recomendações de atividade física (por exemplo, o guia da OMS e dos EUA), o Guia de Atividade Física para a População Brasileira não é um documento pétreo. Logo, precisará ser avaliado e constantemente atualizado com vistas a superar suas limitações e cumprir seu papel de contribuir para promoção da atividade física na população brasileira.

\section{Conflito de interesse}

Os autores declaram não haver conflito de interesse.

\section{Financiamento}

O presente trabalho foi financiado pelo Ministério da Saúde do Brasil a partir do desenvolvimento do Guia Brasileiro de Ativida- 
de Física para População Brasileira, por meio do Termo de Execução Descentralizada n ${ }^{\circ}$ 56/2019 (projeto: 79224219002/2019; processo: 25000.171034/2019-27).

\section{Contribuição dos autores}

Dumith SC, Prazeres Filho A, Cureau FV, Farias Júnior JC, Mello JB, Silva MP, Matias TS, Lopes WA participaram da concepção do manuscrito, coleta e análise dos dados e redação do manuscrito. Magalhães LL participou da coleta dos dados e redação do manuscrito. Hallal PC participou da concepção, redação e revisão geral do manuscrito.

\section{Agradecimentos}

Os autores agradecem aos jovens, pais, professores e gestores que contribuíram para a construção deste capítulo do Guia.

\section{Referências}

1. Bull FC, Al-Ansari SS, Biddle S, Borodulin K, Buman MP, Cardon G, et al. World Health Organization 2020 guidelines on physical activity and sedentary behaviour. Br J Sports Med. 2020;54(24):1451-62.

2. Powell KE, King AC, Buchner DM, Campbell WW, DiPietro L, Erickson KI, et al. The scientific foundation for the physical activity guidelines for Americans, 2nd edition. J Phys Act Health. 2019;16(1):1-11.

3. U.S. Department Of Health and Human Services. Physical Activity Guidelines for Americans, 2 nd edition. Washington, DC; 2018 .

4. Leech RM, McNaughton SA, Timperio A. The clustering of diet, physical activity and sedentary behavior in children and adolescents: a review. Int J Behav Nutr Phys Act. 2014;11(1):4.

5. Paavola M, Vartiainen E, Haukkala A. Smoking, alcohol use, and physical activity: a 13-year longitudinal study ranging from adolescence into adulthood. J Adolesc Heal. 2004;35(3):238-44.

6. Hallal PC, Andersen LB, Bull FC, Guthold R, Haskell W, Ekelund U, et al. Global physical activity levels: surveillance progress, pitfalls, and prospects. Lancet. 2012;380(9838):247-57.

7. Guthold R, Stevens GA, Riley LM, Bull FC. Global trends in insufficient physical activity among adolescents: a pooled analysis of 298 population-based surveys with $1 \cdot 6$ million participants. Lancet Child Adolesc Heal. 2020;4(1):23-35.

8. Condessa LA, Soares CA, Mielke GI, Malta DC, Caiaffa WT. Prevalence of physically active adolescents in Brazilian capitals: National Adolescent School-based Health Survey 2012 and 2015. Rev Bras Epidemiol. 2018;21(suppl 1):e180012.

9. Australian Government Department of H. Australian 24Hour Movement Guidelines for Children (5-12 years) and Young People (13-17 years): An Integration of Physical Activity, Sedentary Behaviour, and Sleep. Canberra; 2017.

10. Chen P, Wang D, Shen H, Yu L, Gao Q, Mao L, et al. Physical activity and health in Chinese children and adolescents: expert consensus statement (2020). Br J Sports Med. 2020;54(22):1321-31.

11. Piercy KL, Troiano RP, Ballard RM, Carlson SA, Fulton JE, Galuska DA, et al. The Physical Activity Guidelines for Americans. JAMA. 2018;320(19):2020-8.
12. Tremblay MS, Carson V, Chaput JP, Connor Gorber S, Dinh T, Duggan M, et al. Canadian 24-hour movement guidelines for children and youth: An integration of physical activity, sedentary behaviour, and sleep. Appl Physiol Nutr Metab. 2016;41(6):S311-S27.

13. Weggemans RM, Backx FJG, Borghouts L, Chinapaw M, Hopman MTE, Koster A, et al. The 2017 Dutch Physical Activity Guidelines. Int J Behav Nutr Phys Act. 2018;15(1):58.

14. Varela AR, Pratt M, Powell K, Lee IM, Bauman A, Heath G, et al. Worldwide surveillance, policy, and research on physical activity and health: the global observatory for physical activity. J Phys Act Health. 2017;14(9):701-9.

15. World Health Organization. WHO Guidelines on Physical Activity and Sedentary Behaviour for Children and Adolescents, Adults and Older Adults. Geneva; 2020. 104 p.

16. The Canadian Society for Exercise P. Canadian 24Hour Movement Guidelines for Children and Youth: An Integration of Physical Activity, Sedentary Behaviour, and Sleep. Ottawa; 2016. 2 p.

17. United Kingdom. UK Chief Medical Officers' Physical Activity Guidelines. 2019.

18. Ministerio De Salud, Secretaria Nacional Del Desporte. ¡A MOVERSE! Guía de actividad física. Montevidéu; 2017.

19. Ministerio de Salud de la Nación. Manual Director de Actividad Fisica y Salud de la Republica Argentina. Buenos Aires; 2013.116 p.

20. Federal Office of Sport, Federal Office of Public Health, Health Promotion Switzerland, Swiss Council for Accident Prevention, Swiss Accident insurance Fund, Health and Physical Activity Network Switzerland. Health-Enhancing Physical Activity. Magglingen; 2013. 28 p.

21. Health Council of the Netherlands. Physical activity guidelines 2017. Amsterdam: 2017. 45 p.

22. Ministerio de Sanidad, Servicios Sociales e Igualdad. Actividad Física para la Salud y Reducción del Sedentarismo. Recomendaciones para la población. Estrategia de Promoción de la Salud y Prevención en el SNS. Madrid; 2015.

23. The State of Qatar. The State of Qatar National Physical Activity Guidelines First Edition. Doha; 2014.70 p.

24. Faigenbaum AD, MacDonald JP, Stracciolini A, Rebullido TR. Making a strong case for prioritizing muscular fitness in youth physical activity guidelines. Curr Sports Med Rep. 2020;19(12):530-6.

25. Poitras VJ, Gray CE, Borghese MM, Carson V, Chaput J-P, Janssen I, et al. Systematic review of the relationships between objectively measured physical activity and health indicators in school-aged children and youth. Appl Physiol Nutr Metab. 2016;41(6 (Suppl. 3)):S197-S239.

26. García-Hermoso A, Alonso-Martínez AM, Ramírez-Vélez R, Pérez-Sousa MÁ, Ramírez-Campillo R, Izquierdo M. Association of physical education with improvement of health-related physical fitness outcomes and fundamental motor skills among youths: a systematic review and metaanalysis. JAMA Pediatr. 2020;174(6):e200223

27. Chaput J-P, Willumsen J, Bull F, Chou R, Ekelund U, Firth J, et al. $2020 \mathrm{WHO}$ guidelines on physical activity and sedentary behaviour for children and adolescents aged 5-17 years: summary of the evidence. Int J Behav Nutr Phys Act. 2020;17(1):141.

28. Sallis JF, Cervero RB, Ascher W, Henderson KA, Kraft MK, Kerr J. An Ecological approach to creating active living communities. Annu Rev Public Health. 2006;27(1):297-322. 
29. Mendonça G, Cheng LA, Mélo EN, Farias Júnior JC.

Recebido: 03/02/2021 Physical activity and social support in adolescents: A systematic review. Health Educ Res. 2014;29(5):822-39.

Aprovado: 06/04/2021

30. Umstattd Meyer MR, Bridges CN, Schmid TL, Hecht

AA, Pollack Porter KM. Systematic review of how Play Streets impact opportunities for active play, physical activity, neighborhoods, and communities. BMC Public Health. 2019;19(1):335.

\section{Como citar este artigo:}

Dumith SC, Prazeres Filho A, Cureau FV, Farias Júnior JC, Mello JB, Silva MP, Matias TS, Lopes WA, Magalhães LL, Hallal PC. Atividade física para crianças e jovens: Guia de Atividade Física para a População Brasileira. Rev Bras Ativ Fís Saúde. 2021;26:e0214. DOI: 10.12820/

$$
\text { rbafs. } 26 e 0214
$$

